\title{
Genetic stock structure of the southern African hakes Merluccius capensis and M.paradoxus
}

\author{
W. S. Grant ${ }^{1}$ * , R. W. Leslie ${ }^{2}$ I. I. Becker ${ }^{1}$ \\ ${ }^{1}$ Department of Microbiology, University of Cape Town, Rondebosch 7700, South Africa \\ ${ }^{2}$ Sea Fisheries Research Institute, Private Bag X2, Rogge Bay 8012, South Africa
}

\begin{abstract}
We estimated the genetic components of geographic variation among stocks of 2 sympatric southern African hakes, Merluccius capensis and M. paradoxus, using allozyme population markers detected by protein electrophoresis. In M. capensis 6 of 33 protein-coding loci were sufficiently polymorphic to test hypotheses of genetic stock structure. Significant but unexplained allele frequency differences were detected between sexes at 5 locations. Significant allele frequency differences between Namibian and South African samples were detected for $C k-A$, indicating at least some genetic subdivision between these regional stocks. The absolute amount of differentiation, however, was small (average genetic distance only 0.0008). On average over loci, $98.3 \%$ of the total gene diversity was contained within samples, $0.7 \%$ was due to differences between sexes, $0.7 \%$ to differences among locations within regions, and $0.3 \%$ to differences between regions. In $M$. paradoxus significant allele frequency differences were detected for $\mathrm{Mpi}$ among the South African samples, but the amount of differentiation between samples was very small. There were no significant allele frequency differences between Namibian and South African samples. Most of the gene diversity $(98.7 \%$ ) was contained on average within samples, $0.3 \%$ was due to differences between sexes, $0.2 \%$ to differences between locations within regions and $0.3 \%$ to differences between regions. A review of morphological variation together with the genetic data presented here suggest that both species of hake are minimally subdivided into 2 regional stocks. The most conservative approach to the management of the regional stocks of hake is to manage the Namibian and South African stocks independently of one another
\end{abstract}

\section{INTRODUCTION}

Two sympatric species of hake, Merluccius capensis and M.paradoxus, inhabit the coastal waters of southern Africa and support a large bottom trawl fishery. The former species generally inhabits continental shelf waters to a depth of $440 \mathrm{~m}$, whereas the latter species inhabits deeper waters from 140 to $850 \mathrm{~m}$. The geographic distributions of both fishes are closely associated with the cold water of the northward flowing Benguela Upwelling System on the west coast (Botha 1980, Shannon 1985). M.capensis is distributed between $12^{\circ} \mathrm{S}$ lat. on the west coast of Africa to about $27^{\circ} \mathrm{E}$ long. on the south coast, but $M$. paradoxus has a more restricted geographic distribution between $18^{\circ} \mathrm{S}$ lat. on the west coast to about $31.5^{\circ} \mathrm{E}$ long. on the south coast. Although the cranial morphology (Franca 1954, van Eck 1969, Botha 1971, Bentz 1976) and the genetic profiles (Jones \& Mackie 1970, Grant et al. 1988,

\footnotetext{
- Present address: Department of Genetics, University of the Witwatersrand, Johannesburg 2050, South Africa
}

Becker et al. 1988) of these species are distinct, it is impractical to identify the proportions of each species in partially processed commercial landings.

The 2 species of southern African hakes are harvested by bottom trawl more or less continuously on the west coast from the Agulhas Bank to Walvis Bay depending upon bottom topography. Because of its greater geographic range Merluccius capensis is also fished in the northern part of Namibia. These geographic distributions of fishing effort also reflect the more or less continuous distributions of the 2 species (Macpherson et al. 1986, Payne et al. 1986). Concentrations of larvae and juveniles, however, are more patchy. In $M$. capensis, high concentrations of larvae and juveniles are generally found off the Orange River and to the south, but the centers of these concentrations vary from year to year. On the other hand, larvae and juveniles of $M$. paradoxus are found off southern Namibia and off Cape Point in more offshore waters (Macpherson et al. 1986, Payne et al. 1986). The degree to which these concentrations of larvae and juveniles are isolated from one another is not known. 
One important consideration in the commercial exploitation of a fishery resource is the identification of any discrete population or stock units that might exist. These idealized stock units are usually thought of as self-maintaining groups of fish that are temporally or spatially isolated from one another and because of their isolation are generally thought to be genetically distinct to some degree or another (Ricker 1972, Booke 1981). Reproductive isolation between stocks of marine fishes may arise by homing to different spawning areas (e.g. Pacific herring Clupea pallasi, Hourston 1982) or by hydrographic features which reduce or prevent migration between areas. For example, Iles \& Sinclair (1982) have proposed that stocks of Atlantic herring Clupea harengus are structured at the larval stage by oceanographic gyres which create stable larval-retention areas.

Several methods have been employed to measure the amount of relatedness between stocks. Tagging is one method, which yields direct evidence of migration (e.g. Haugen et al. 1969), but cannot be applied to hakes because few fish survive the transit to the surface (Jones 1974). Other approaches are to define stocks (e.g. salmon) by geographic differences in body morphology or meristic characters or by the analyses of otoliths (Ihssen et al. 1981). But these methods depend upon finding pronounced environmental differences between areas, a situation that does not occur in the range of southern African hakes. None of these techniques, however, yields unequivocal data on the genetic relatedness between stocks. In the present study we have chosen protein electrophoresis to estimate the genetic component of geographic variation in the southern African hakes (Allendorf \& Utter 1979). We assume that electrophoretic variants are neutral or nearly neutral to the effects of natural selection and thus largely reflect the historical effects of population size and migration (Lewontin 1974).

There have been few genetic studies of other species of hake in the genus Merluccius. One electrophoretic study of the Pacific hake ( $M$. productus), however, demonstrated the presence of allele-frequency differences between populations located in Puget Sound, a large marine embayment, and offshore oceanic populations (Utter \& Hodgins 1969, 1971, Utter et al. 1970). Another study of the North Atlantic hake ( $M$. merluccius), however, failed to detect any genetic differentiation among populations (Mangaly \& Jamieson 1978)

The purpose of this study was to investigate the genetic relationship between Namibian and South African stocks of the 2 species of hake. In our experimental design we subdivided our samples for each species into 2 groups that more or less concided with the political boundary between Namibia and South
Africa because this subdivision also appeared to reflect regional differences in morphology. There is a large potential for movement between locations at different life-history stages for each of these species. Fish spawn offshore in deep water in spring, and pelagic eggs and larvae drift in currents near the surface in the thermocline. Juveniles begin to lead a demersal existence during their first year (Botha 1973). Although extensive seasonal, longshore migration characterizes other species of hake (Bailey et al. 1982), the amount of migration in the 2 southern African species is uncertain. Botha (1980) argued from annual catch data that there was no strong seasonal patterns of migration in the Cape of Good Hope area. There is, however, some evidence of seasonal migration in Namibian waters (Inada 1981, Anon. 1986).

\section{MATERIALS AND METHODS}

Tissue samples or whole fish of Merluccius capensis (13 locations) and $M$. paradoxus (10 locations) were collected from coastal waters extending from northern Namibia to the south coast of South Africa (Table 1; Fig. 1). Although the morphologies of these species are superficially very similar, they can be identified by differences in the gill arch tubercles (van Eck 1969). Samples were frozen and held at $-25^{\circ} \mathrm{C}$ until laboratory analyses.

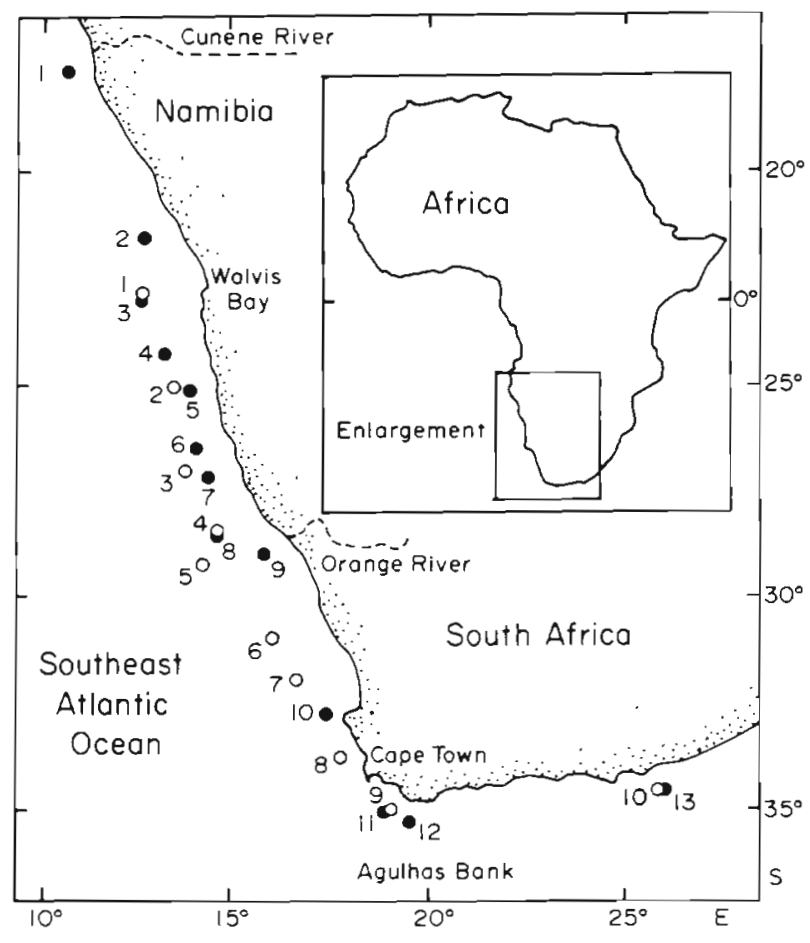

Fig. 1 Sampling locations of Merluccius capensis ( $\bullet$ ) and $M$. paradoxus (o) used in study of genetic variation 
Loci were designated using evolutionary nomenclature inferred from tissue distributions of gene expression (e.g. Markert et al. 1975, Fisher et al. 1980, Frick 1983). Otherwise, loci encoding functionally similar proteins were numbered by the mobilities of their products beginning from the cathodal end of a gel. Alleles for each species were designated by their electrophoretic mobilities relative to the most common allele for that species, which was arbitrarily designated 100. Alleles migrating cathodally from the origin were prefixed with a minus sign. The names of the loci examined, their Enzyme Comission numbers, buffers and tissues giving the best results are presented in Table 2 .

\section{RESULTS}

\section{Merluccius capensis}

Table 3 presents the allele frequency data for 13 polymorphic loci. Six loci, Ck-A, Gpi-A, Gpi-B, Pgm-A,
$P e p-B$ and $P e p-D$, were sufficiently polymorphic to test hypotheses of population genetic structure. Individuals were sexed in Samples 1, 2, 4, and 6 to 12. Graphical representations of the frequencies of the most-common alleles for polymorphic loci along with $95 \%$ confidence intervals are presented in Fig. 2.

We used the G-test for goodness of fit (Sokal \& Rohlf 1981) to test for departures from Hardy-Weinberg proportions. Genotypic frequencies of loci having rare genotypes were pooled according to Swofford \& Selander (1981). Significant departures from Hardy-Weinberg expectations were detected for $C k-A$ among males of Sample $11\left(G_{1}=5.240, \mathrm{p}<0.05\right)$ and among pooled individuals in this sample $\left(G_{1}=6.07, \mathrm{p}<0.05\right)$. Significant departures were detected for $P e p-B$ in Sample 1 (females $G_{1}=4.32, \mathrm{p}<0.05$; pooled over sexes $G_{1}=4.22, \mathrm{p}<0.05$ ) and in Sample 8 (males $G_{1}=5.94$, $\mathrm{p}<0.05$; pooled over sexes $G_{1}=9.61, \mathrm{p}<0.01$ ). For Pep- $D$, significant departures appeared in Sample 9 (males $G_{1}=5.31, \mathrm{p}<0.05 ;$ pooled over sexes $G_{1}=$ $11.04, \mathrm{p}<0.01$ ) and in Sample 10 (males $G_{1}=10.33$,

Table 1. Merluccius capensis and M. paradoxus. Locations, dates, depths and sizes of samples used for electrophoretic analyses

\begin{tabular}{|c|c|c|c|c|c|c|}
\hline \multirow[t]{2}{*}{ Sample no. } & \multirow[t]{2}{*}{ Location } & \multirow[t]{2}{*}{ Date collected } & \multirow[t]{2}{*}{ Depth $(\mathrm{m})$} & \multicolumn{3}{|c|}{ Sample size } \\
\hline & & & & Total & Male & Female \\
\hline \multicolumn{7}{|c|}{ Merluccius capensis } \\
\hline \multicolumn{7}{|l|}{ Namibia } \\
\hline 1. & $19^{\circ} 42^{\prime} \mathrm{S}, 11^{\circ} 48^{\prime} \mathrm{E}$ & 16 Jan 86 & 195 & 64 & 26 & 38 \\
\hline 2. & $22^{\circ} 03^{\prime} \mathrm{S}, 13^{\circ} 31^{\prime} \mathrm{E}$ & $28 \mathrm{Jan} 86$ & 136 & 1000 & 50 & 50 \\
\hline 3. & $23^{\circ} 02^{\prime} \mathrm{S}, 13^{\circ} 05^{\prime} \mathrm{E}$ & $14 \operatorname{Jan} 84$ & 384 & 94 & & \\
\hline 4. & $24^{\circ} 29^{\prime} \mathrm{S}, 13^{\circ} 36^{\prime} \mathrm{E}$ & 31 Jan 84 & 350 & 85 & 46 & 39 \\
\hline 5. & $25^{\circ} 01^{\prime} \mathrm{S}, 14^{\circ} 06^{\prime} \mathrm{E}$ & $17 \operatorname{Jan} 84$ & 179 & 101 & & \\
\hline 6. & $26^{\circ} 07^{\prime} \mathrm{S}, 14^{\circ} 17^{\prime} \mathrm{E}$ & $18 \mathrm{Jan} 84$ & 216 & 100 & 46 & 54 \\
\hline 7. & $27^{\circ} 02^{\prime} \mathrm{S}, 14^{\circ} 35^{\prime} \mathrm{E}$ & 19 Jan 84 & 298 & 100 & 42 & 58 \\
\hline 8. & $27^{\circ} 57^{\prime} \mathrm{S}, 14^{\circ} 58^{\prime} \mathrm{E}$ & 20 Jan 84 & 193 & 91 & 36 & 55 \\
\hline \multicolumn{7}{|l|}{ South Africa } \\
\hline 9. & $28^{\circ} 52^{\prime} \mathrm{S}, 16^{\circ} 28^{\prime} \mathrm{E}$ & 24 Jan 84 & 86 & 100 & 28 & 72 \\
\hline 10. & $32^{\circ} 55^{\prime} \mathrm{S}, 17^{\circ} 40^{\prime} \mathrm{E}$ & 12 Jan 84 & 301 & 101 & 51 & 50 \\
\hline 11. & $35^{\circ} 15^{\prime} \mathrm{S}, 19^{\circ} 25^{\prime} \mathrm{E}$ & $07 \mathrm{Jan} 84$ & 303 & 100 & 62 & 38 \\
\hline 12 . & $35^{\circ} 00^{\prime} \mathrm{S}, 19^{\circ} 05^{\prime} \mathrm{E}$ & 26 Nov 84 & 150 & 50 & 24 & 26 \\
\hline 13. & $34^{\circ} 20^{\prime} \mathrm{S}, 25^{\circ} 40^{\prime} \mathrm{E}$ & 29 Nov 84 & 205 & 50 & & \\
\hline \multicolumn{7}{|c|}{ Merluccius paradoxus } \\
\hline \multicolumn{7}{|l|}{ Namibia } \\
\hline 1. & $23^{\circ} 02^{\prime} \mathrm{S}, 13^{\circ} 05^{\prime} \mathrm{E}$ & $14 \mathrm{Jan} 84$ & 384 & 89 & & \\
\hline 2. & $25^{\circ} 08^{\prime} \mathrm{S}, 13^{\circ} 38^{\prime} \mathrm{E}$ & $29 \operatorname{Jan} 84$ & 450 & 98 & & \\
\hline 3. & $27^{\circ} 03^{\prime} \mathrm{S}, 14^{\circ} 08^{\prime} \mathrm{E}$ & $28 \mathrm{Jan} 84$ & 420 & 76 & & \\
\hline 4. & $27^{\circ} 57^{\prime} \mathrm{S}, 14^{\circ} 58^{\prime} \mathrm{E}$ & $20 \mathrm{Jan} 84$ & 130 & 100 & & \\
\hline \multicolumn{7}{|l|}{ South Africa } \\
\hline 5. & $29^{\circ} 01^{\prime} \mathrm{S}, 14^{\circ} 36^{\prime} \mathrm{E}$ & $22 \operatorname{Jan} 84$ & 299 & 99 & 58 & 41 \\
\hline 6. & $31^{\circ} 30^{\prime} \mathrm{S}, 16^{\circ} 00^{\prime} \mathrm{E}$ & $15 \mathrm{Jan} 84$ & 478 & 82 & 26 & 56 \\
\hline 7. & $32^{\circ} 05^{\prime} \mathrm{S}, 16^{\circ} 40^{\prime} \mathrm{E}$ & $14 \mathrm{Jan} 84$ & 346 & 94 & 38 & 56 \\
\hline 8. & $33^{\circ} 40^{\prime} \mathrm{S}, 17^{\circ} 30^{\prime} \mathrm{E}$ & $28 \mathrm{Jan} 84$ & 357 & 98 & 41 & 57 \\
\hline 9. & $34^{\circ} 40^{\prime} \mathrm{S}, 18^{\circ} 25^{\prime} \mathrm{E}$ & $09 \operatorname{Jan} 84$ & 305 & 101 & & \\
\hline 10. & $34^{\circ} 20^{\prime} \mathrm{S}, 25^{\circ} 40^{\prime} \mathrm{E}$ & $27 \operatorname{Jan} 84$ & 205 & 46 & & \\
\hline
\end{tabular}


Table 2. Enzymatic proteins (Enzyme Commission numbers), locus abbreviations, tissues with strongest expression, and electrophoretic buffers used in study of southem African hakes. Tissues: $M$, skeletal muscle; $H_{1}$ heart muscle; $L_{1}$ liver; $E_{1}$ eye fluids. Buffer components: (1) Gel: 0.03M Tris, 0.005M citric acid, 0.0006M lithium hydroxide, 0.003M boric acid (pH 8.5); electrode: $0.06 \mathrm{M}$ lithium hydroxide, $0.3 \mathrm{M}$ boric acid (pH 8.1). (2) Gel: 1:4 dilution of electrode buffer; electrode: $0.18 \mathrm{M}$ Tris, $0.1 \mathrm{M}$ boric acid, 0.004M NaEDTA. (3) Gel: $1: 14$ dilution of electrode solution; electrode: $0.15 \mathrm{M}$ Tris, 0.05M citric acid (pH 6.9). (4) Buffer 2 but with addition of $50 \mathrm{mg}$ NAD to the gel and $75 \mathrm{mg}$ NAD to the cathodal electrode compartment

\begin{tabular}{|c|c|c|c|}
\hline Protein & Locus & Tissue (s) & Buffer \\
\hline \multirow[t]{2}{*}{ Creatine kinase (2.7.3.2) } & $C k-A$ & M & 1 \\
\hline & $C k-B$ & $E, M$ & 1 \\
\hline \multirow[t]{2}{*}{ Glucosephosphate isomerase (5.3.1.9) } & Gpi-A & M & 1 \\
\hline & $G p i-B$ & M & 1 \\
\hline \multirow[t]{2}{*}{ Glyceraldehyde phosphate dehydrogenase (1.2.1.12) } & Gap-1 & M & 4 \\
\hline & Gap-2 & $E$ & 4 \\
\hline \multirow[t]{3}{*}{ Glycerol-3-phosphate dehydrogenase (1.1.1.8) } & $G p d-1$ & M & 3 \\
\hline & $G p d-2$ & M & 3 \\
\hline & $G p d-3$ & M & 3 \\
\hline Guanine deaminase $(3.5,4.3)$ & Gda & $\mathrm{H}, \mathrm{L}$ & 2 \\
\hline Isocitrate dehydrogenase (1.1.1.42) & $I d h-A$ & $\mathrm{M}, \mathrm{H}$ & 3 \\
\hline \multirow[t]{3}{*}{ Lactate dehydrogenase (1.1.1.27) } & $L d h-A$ & M & 1 \\
\hline & $L d h-B$ & M & 1 \\
\hline & $L d h-C$ & $\mathrm{~L}$ & 1 \\
\hline \multirow[t]{2}{*}{ Malate dehydrogenase (1.1.1.37) } & $M d h-A$ & M & 3 \\
\hline & $M d h-B$ & M & 3 \\
\hline Malic enzyme (1.1.1.40) & $M e$ & $\mathrm{~L}, \mathrm{M}$ & 3 \\
\hline Mannosephosphate isomerase (5.3.1.8) & Mpi & $\mathrm{H}, \mathrm{M}$ & 2 \\
\hline Nucleoside phosphorylase (2.4.21) & $N p$ & $E$ & 2 \\
\hline \multirow[t]{5}{*}{ Peptidase $(3.4 .11)$} & Pep-A $A^{1,2}$ & $\mathrm{M}, \mathrm{H}$ & 2 \\
\hline & $P e p-B^{3}$ & M & 2 \\
\hline & Pep-C $C^{1}$ & M & 2 \\
\hline & $P e p-D^{4}$ & $M$ & 2 \\
\hline & $P e p-X^{5}$ & M & 2 \\
\hline \multirow[t]{2}{*}{ Phosphoglucomutase $(2.7 .5 .1)$} & $P g m-A$ & M & 1 \\
\hline & $P g m-B$ & $\mathrm{M}, \mathrm{L}$ & 1 \\
\hline Phosphogluconate dehydrogenase $(1.1 .1 .44)$ & Pgd & $M$ & 3 \\
\hline \multirow[t]{5}{*}{ Protein (non-specific) } & Pt-1 & M & 1 \\
\hline & $P_{t-2}$ & M & 1 \\
\hline & $P_{t-3}$ & $M$ & 1 \\
\hline & $P_{t-4}$ & $M$ & 1 \\
\hline & Pt-5 & $\mathrm{M}$ & 1 \\
\hline Superoxide dismutase $(1.15 .1 .1)$ & Sod-1 & $\mathrm{H}, \mathrm{L}$ & 1 \\
\hline Sorbitol dehydrogenase (1.1.1.14) & $S d h$ & $\mathrm{~L}$ & 1 \\
\hline \multicolumn{4}{|l|}{${ }^{2}$ Substrate: leucyl-tyrosine } \\
\hline \multicolumn{4}{|l|}{${ }^{2}$ Not resolved for routine scoring } \\
\hline \multicolumn{4}{|l|}{${ }^{3}$ Substrate: leucyl-glycyl-glycine } \\
\hline \multicolumn{4}{|l|}{${ }^{4}$ Substrate: phenylananine-proline } \\
\hline${ }^{5}$ Substrate: appeared using leu-tyr and leu-gly-gly & & & \\
\hline
\end{tabular}

$\mathrm{p}<0.01 ;$ pooled over sexes $\left.G_{1}=9.01, \mathrm{p}<0.01\right)$. A significant departure was also detected for $P g m-A$ in Sample $5\left(G_{1}=6.38, \mathrm{p}<0.05\right)$.

We used a hierarchical analysis of allele frequencies with the G-test for independence (Sokal \& Rohlf 1981) to test for heterogeneity between sexes in each sample at the lowest level of population organization, among locations within regions, and between regions (northern or Namibia and southern or South Africa) at the highest nested level. The sums of the $G$ values and their associated degrees of freedom over loci give summary statistics for each comparison. At the lowest level of population structure, significant allele frequency differences between sexes appeared in Sample $2\left(\right.$ Pep- $\left.B_{1} G_{1}=5.74, \mathrm{p}<0.05\right)$, Sample $7 \mathrm{Ck}-A_{1} G_{1}=$ 7.61, $\mathrm{p}<0.01)$, Sample $9\left(C k-A, G_{1}=5.47, \mathrm{p}<0.05\right)$ and Sample $10\left(\right.$ Gpi-B, $G_{1}=5.37, \mathrm{p}<0.05 ; P g m-A, G_{1}$ $=5.50, \mathrm{p}<0.05$ ).

There was no significant allele-frequency heterogeneity among Samples 10 to 13 from the southern region. On the other hand, we detected significant heterogeneity among Samples 1 to 9 from the northem region for $G$ pi-A $\left(G_{16}=28.49, \mathrm{p}<0.05\right)$. At the highest nested level, there was a highly significant degree of 
Table 3. Merluccius capensis. Allelic frequencies. The following loci were fixed for the 100 allele: $C k-B, G a p-1, G p d-A, G p d-B$, Gpd-C, Ldh-C, Mdh-B, Mpi, Np, Pep-X (leu-tyr), Pgd, Pt-1, Pt-2, Pt-3, Pt-4, Pt-5, Sdh, Sod

\begin{tabular}{|c|c|c|c|c|c|c|c|c|c|c|c|c|c|c|}
\hline \multirow[t]{2}{*}{ Locus } & \multirow[t]{2}{*}{ Allele } & \multicolumn{13}{|c|}{ Sample number } \\
\hline & & 1 & 2 & 3 & 4 & 5 & 6 & 7 & 8 & 9 & 10 & 11 & 12 & 13 \\
\hline \multirow[t]{6}{*}{$C k-A$} & 150 & - & - & - & - & - & - & 0.010 & - & - & - & - & - & - \\
\hline & 100 & 0.812 & 0.850 & 0.799 & 0.847 & 0.837 & 0.862 & 0.790 & 0.800 & 0.806 & 0.696 & 0.750 & 0.740 & 0.710 \\
\hline & 96 & - & - & - & - & 0.005 & 0.040 & - & - & - & - & - & - & - \\
\hline & 85 & 0.188 & 0.150 & 0.196 & 0.153 & 0.158 & 0.138 & 0.160 & 0.200 & 0.194 & 0.304 & 0.250 & 0.260 & 0.290 \\
\hline & 70 & - & - & 0.006 & - & - & - & - & - & - & - & - & - & - \\
\hline & $2 \mathrm{~N}$ & 128 & 200 & 184 & 170 & 196 & 196 & 200 & 170 & 196 & 194 & 200 & 100 & 100 \\
\hline \multirow[t]{3}{*}{$G d a$} & 100 & 1.000 & 1.000 & 1.000 & 1.000 & 1.000 & 1.000 & 1.000 & 1.000 & 1.000 & 0.995 & 1.000 & 1.000 & 1.000 \\
\hline & 90 & - & - & - & - & - & - & - & - & - & 0.005 & - & - & - \\
\hline & $2 \mathrm{~N}$ & 128 & 200 & 188 & 170 & 202 & 200 & 200 & 182 & 200 & 202 & 200 & 100 & 100 \\
\hline \multirow[t]{6}{*}{$G p i-A$} & 250 & - & - & 0.005 & - & - & 0.005 & - & 0.005 & - & - & - & - & 0.010 \\
\hline & 100 & 0.242 & 0.145 & 0.258 & 0.159 & 0.248 & 0.160 & 0.190 & 0.132 & 0.170 & 0.198 & 0.237 & 0.160 & 0.150 \\
\hline & -80 & - & - & - & 0.006 & - & - & - & - & - & - & - & - & - \\
\hline & -100 & 0.671 & 0.805 & 0.688 & 0.771 & 0.708 & 0.760 & 0.770 & 0.791 & 0.740 & 0.767 & 0.707 & 0.800 & 0.810 \\
\hline & -200 & 0.047 & 0.050 & 0.048 & 0.065 & 0.040 & 0.075 & 0.040 & 0.071 & 0.075 & 0.035 & 0.056 & 0.040 & 0.020 \\
\hline & $2 \mathrm{~N}$ & 128 & 200 & 186 & 170 & 202 & 200 & 200 & 182 & 200 & 202 & 198 & 100 & 100 \\
\hline \multirow[t]{8}{*}{$G p i-B$} & 110 & - & - & - & - & 0.010 & - & - & - & - & - & - & - & - \\
\hline & 100 & 0.984 & 0.950 & 0.952 & 0.929 & 0.931 & 0.985 & 0.955 & 0.945 & 0.945 & 0.965 & 0.965 & 0.950 & 0.990 \\
\hline & 90 & - & 0.005 & - & - & - & - & - & - & - & - & - & - & - \\
\hline & 83 & 0.016 & 0.030 & 0.027 & 0.047 & 0.050 & 0.010 & 0.045 & 0.038 & 0.030 & 0.015 & 0.010 & 0.020 & - \\
\hline & 75 & - & - & - & - & - & 0.005 & - & - & - & - & - & - & - \\
\hline & 70 & - & 0.021 & 0.022 & 0.010 & - & - & - & 0.016 & 0.025 & 0.020 & 0.025 & 0.030 & 0.010 \\
\hline & 60 & - & 0.005 & - & - & - & - & - & - & - & - & - & - & - \\
\hline & $2 N$ & 128 & 200 & 186 & 170 & 202 & 200 & 200 & 182 & 200 & 202 & 200 & 100 & 100 \\
\hline \multirow[t]{3}{*}{$I d h-A$} & 10 & 1.000 & 1.000 & 1.000 & 0.987 & 0.994 & 1.000 & 1.000 & 1.000 & 1.000 & 1.000 & 1.000 & 1.000 & 1.000 \\
\hline & 85 & - & - & - & 0.013 & 0.006 & - & - & - & - & - & - & - & - \\
\hline & $2 \mathrm{~N}$ & 128 & 200 & 188 & 170 & 202 & 200 & 200 & 182 & 200 & 202 & 200 & 100 & 100 \\
\hline$L d h-A$ & 100 & 1.000 & 0.995 & 1.000 & 1.000 & 1.000 & 1.000 & 1.000 & 1.000 & 1.000 & 1.000 & 1.000 & 1.000 & 1.000 \\
\hline & -200 & - & 0.005 & - & - & - & - & - & - & - & - & - & - & - \\
\hline & $2 \mathrm{~N}$ & 128 & 200 & 188 & 170 & 202 & 200 & 200 & 182 & 200 & 202 & 200 & 100 & 100 \\
\hline$L d h-B$ & 100 & 1.000 & 1.000 & 1.000 & 1.000 & 1.000 & 0.990 & 0.995 & 1.000 & 1.000 & 0.985 & 0.995 & 0.990 & 0.980 \\
\hline & 70 & - & - & - & - & - & 0.010 & 0.005 & - & - & 0.015 & 0.005 & 0.010 & 0.020 \\
\hline & $2 \mathrm{~N}$ & 128 & 200 & 188 & 170 & 202 & 200 & 200 & 182 & 200 & 202 & 200 & 100 & 100 \\
\hline$M d h-A$ & 180 & - & - & - & 0.006 & - & - & - & - & - & - & - & - & - \\
\hline & 100 & 1.000 & 1.000 & 0.989 & 0.988 & 1.000 & 1.000 & 1.000 & 1.000 & 1.000 & 1.000 & 1.000 & 1.000 & 1.000 \\
\hline & 50 & - & - & - & 0.006 & - & - & - & - & - & - & - & - & - \\
\hline & $2 \mathrm{~N}$ & 128 & 200 & 188 & 170 & 202 & 200 & 200 & 182 & 200 & 202 & 200 & 100 & 100 \\
\hline $\mathrm{Me}$ & 105 & 1.000 & 1.000 & 0.987 & 1.000 & 1.000 & 1.000 & 1.000 & 1.000 & 1.000 & 1.000 & 1.000 & 0.980 & 0.990 \\
\hline & 100 & - & - & 0.013 & - & - & - & - & - & - & - & - & 0.020 & 0.010 \\
\hline & $2 \mathrm{~N}$ & 128 & 200 & 188 & 170 & 202 & 200 & 200 & 182 & 200 & 202 & 200 & 100 & 100 \\
\hline$P e p-D$ & 103 & - & - & 0.006 & 0.013 & - & - & 0.010 & - & - & 0.020 & 0.036 & 0.030 & 0.020 \\
\hline & 100 & 0.508 & 0.566 & 0.529 & 0.677 & 0.602 & 0.551 & 0.526 & 0.630 & 0.540 & 0.611 & 0.521 & 0.540 & 0.610 \\
\hline & 94 & 0.062 & 0.030 & 0.092 & 0.038 & 0.051 & 0.081 & 0.088 & 0.032 & 0.070 & 0.040 & 0.089 & 0.010 & - \\
\hline & 90 & - & - & - & - & - & - & 0.021 & - & 0.005 & - & - & - & - \\
\hline & 85 & 0.422 & 0.394 & 0.374 & 0.272 & 0.347 & 0.369 & 0.356 & 0.312 & 0.380 & 0.323 & 0.354 & 0.410 & 0.360 \\
\hline & 80 & 0.008 & 0.010 & - & - & - & - & - & 0.026 & 0.005 & 0.005 & - & 0.010 & 0.010 \\
\hline & $2 \mathrm{~N}$ & 128 & 198 & 174 & 158 & 196 & 198 & 194 & 154 & 200 & 198 & 192 & 100 & 100 \\
\hline Pep-B & 105 & 0.421 & 0.320 & 0.394 & 0.442 & 0.326 & 0.420 & 0.449 & 0.377 & 0.296 & 0.406 & 0.365 & 0.306 & 0.306 \\
\hline & 100 & 0.563 & 0.660 & 0.606 & 0.569 & 0.613 & 0.570 & 0.546 & 0.565 & 0.679 & 0.589 & 0.609 & 0.612 & 0.592 \\
\hline & 95 & 0.016 & 0.020 & - & 0.009 & 0.062 & 0.010 & 0.005 & 0.058 & 0.015 & 0.005 & 0.026 & 0.082 & 0.102 \\
\hline & 90 & - & - & - & - & - & - & - & - & 0.010 & - & - & - & - \\
\hline & $2 \mathrm{~N}$ & 126 & 200 & 170 & 116 & 194 & 200 & 196 & 154 & 196 & 180 & 192 & 98 & 98 \\
\hline$P g m-A$ & 125 & 0.008 & - & 0.016 & - & - & 0.005 & - & 0.023 & - & 0.005 & - & - & - \\
\hline & 115 & 0.055 & 0.050 & 0.027 & 0.065 & 0.025 & 0.051 & 0.070 & 0.068 & 0.060 & 0.074 & 0.060 & 0.030 & 0.060 \\
\hline & 110 & 0.008 & 0.010 & - & - & 0.010 & - & - & - & 0.005 & - & - & 0.040 & - \\
\hline & 100 & 0.883 & 0.980 & 0.903 & 0.894 & 0.950 & 0.899 & 0.875 & 0.864 & 0.885 & 0.891 & 0.885 & 0.900 & 0.880 \\
\hline & 80 & 0.008 & - & - & 0.012 & 0.005 & - & - & - & 0.005 & - & - & 0.040 & - \\
\hline & 75 & 0.031 & 0.030 & 0.016 & 0.012 & 0.010 & 0.035 & 0.025 & 0.028 & 0.030 & 0.020 & 0.045 & 0.030 & 0.030 \\
\hline & 60 & 0.008 & 0.020 & 0.320 & 0.006 & - & 0.005 & 0.015 & - & 0.015 & 0.005 & - & - & 0.010 \\
\hline & 55 & - & - & 0.005 & 0.012 & - & 0.005 & 0.015 & 0.017 & - & 0.005 & 0.010 & - & 0.010 \\
\hline & $2 \mathrm{~N}$ & 128 & 200 & 186 & 170 & 202 & 198 & 200 & 176 & 200 & 202 & 200 & 100 & 100 \\
\hline$P g m-B$ & 103 & 0.008 & - & - & 0.029 & - & - & - & - & - & - & - & 0.020 & - \\
\hline & 100 & 0.961 & 0.985 & 0.995 & 0.953 & 1.000 & 1.000 & 1.000 & 0.989 & 1.000 & 0.995 & 1.000 & 0.980 & 0.970 \\
\hline & 95 & 0.016 & 0.015 & 0.005 & 0.012 & - & - & - & 0.011 & - & 0.005 & - & - & - \\
\hline & 90 & 0.0008 & - & - & 0.006 & - & - & - & - & - & - & - & - & - \\
\hline & $2 \mathrm{~N}$ & 128 & 200 & 188 & 170 & 202 & 200 & 200 & 182 & 200 & 202 & 200 & 100 & 100 \\
\hline
\end{tabular}




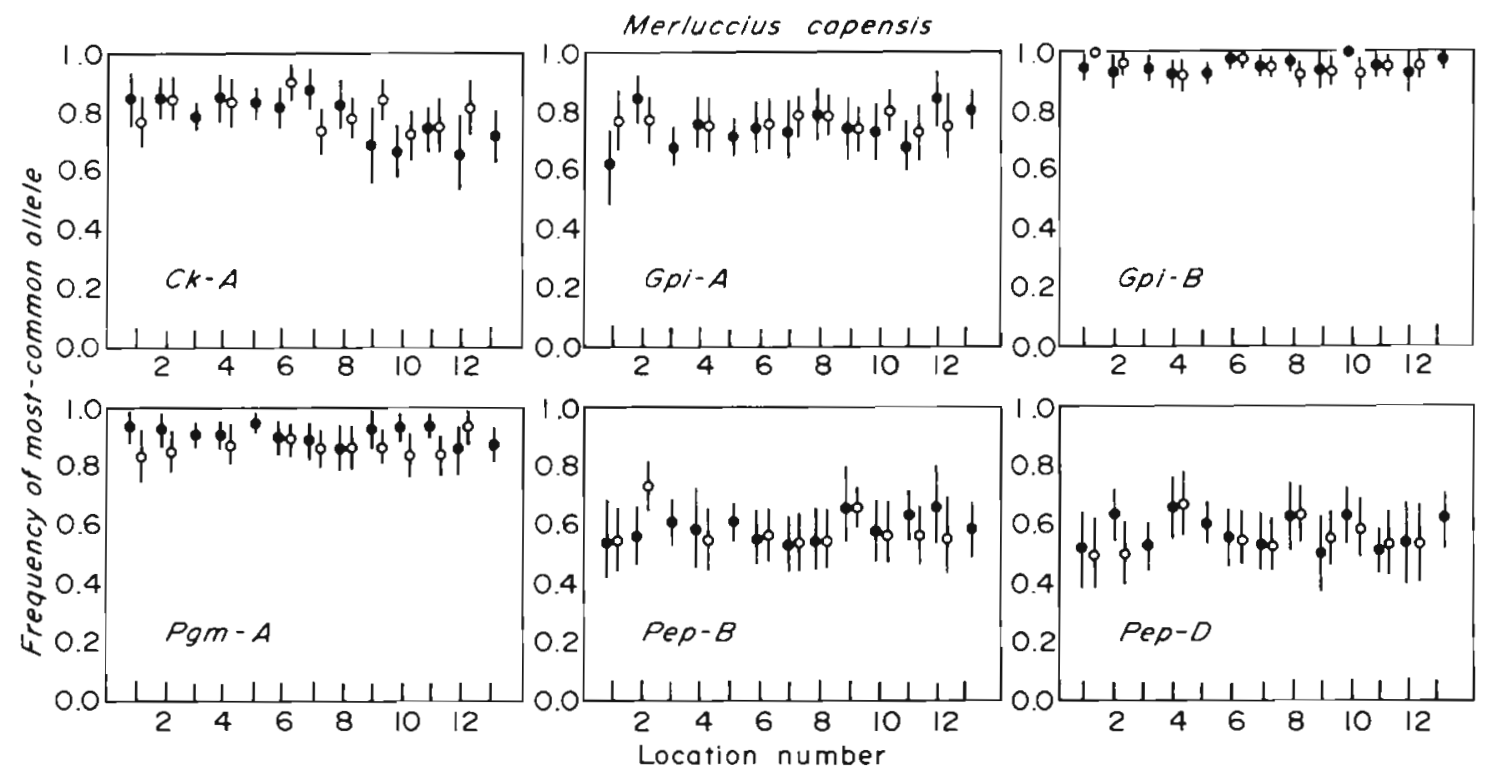

Fig. 2. Merluccius capensis. Frequencies of most common alleles in samples. ( $\bullet$ ) Frequencies of males; (o) frequencies of females. Vertical bars represent 4 binomial standard errors and approximate a $96 \%$ confidence interval. Location numbers as in Table 1 and Fig. 1

heterogeneity between Namibian and South African samples for $C k-A\left(G_{2}=22.32, p<0.001\right)$. The frequencies of the $C k-A^{100}$ allele pooled over locations for these 2 regions were 0.823 and 0.724 , respectively. Although none of the other enzymes showed significance for this comparison, the $G$-statistic summed over all loci was also highly significant $\left(G_{7}=30.92, \mathrm{p}<0.01\right)$.

This heirarchical analysis of allele frequency heterogeneity is not entirely appropriate because significantly different allele frequencies at lower nested levels should not be pooled for comparisons at higher levels. To circumvent this problem we computed an approximate $F$ ratio by dividing the summed $G$ statistics by their degrees of freedom, and comparing the heterogeneity at one nested level with that at the next lower level (Smouse \& Kojima 1972). These results show that the degree of allele-frequency heterogeneity between samples within each of the regions was not greater than the heterogeneity between sexes (Namibia, $F_{56,56}=1.73$, ns; South Africa $F_{21,21}=0.54$, ns). There was, however, a significant degree of heterogeneity between Nambian and South African stocks relative to the degree of heterogeneity among samples within each of these stocks $\left(F_{7.77}=3.186\right.$, $\mathrm{p}<0.01$ )

We used the gene diversity analysis of Nei (1973) with the computing algorithm of Chakraborty et al. (1982) to estimate the relative amounts of gene differentiation between samples at 3 levels of subdivision. Total gene diversity $\left(H_{\mathrm{T}}\right)$ was partitioned into its components where $G_{\mathrm{SP}}=\left(H_{\mathrm{P}}-H_{\mathrm{S}}\right) / H_{\mathrm{T}}$ was the diversity due to differences between sexes ( $S$ ) within populations $(P), G_{\mathrm{PR}}=\left(H_{\mathrm{R}}-H_{\mathrm{P}}\right) / H_{\mathrm{T}}$ was the relative diversity due to differences between populations within regions $(R)$, and $G_{\mathrm{RT}}=\left(H_{\mathrm{T}}=\left(H_{\mathrm{T}}-H_{\mathrm{R}}\right) / H_{\mathrm{T}}\right.$ was the relative diversity due to differences between regions. The residual gene diversity was the proportion due to within-sample variation. These statistics were averaged over loci to yield a summary statistic for each level of population subdivision. Since the sexes of individuals were not identified in all samples, we used an incomplete experimental design at the lowest level of our analysis in which gene diversity was averaged only over those samples for which data were available. This did not affect the rest of the analysis, because the allele-frequency data were pooled for the the computation of gene diversities at the next hierarchical level of population division.

Average sample heterozygosities ranged from 0.054 to 0.070 and averaged 0.063 over all samples. The gene diversity analysis indicated that this within-sample variability represented $98.3 \%$ of the total variability on average over loci. Allele-frequency differences between sexes represented $0.6 \%$, differences between samples within regions represented $0.7 \%$, and differences between regions represented $0.3 \%$ of the total genetic variability. The average genetic distance (Nei 1972 ) between samples from the 13 locations was 0.0006 and a UPGMA cluster analysis (Sneath \& Sokal 1973) revealed no meaningful geographic relationships among the samples.

\section{Merluccius paradoxus}

Allele frequencies of 15 polymorphic loci are presented in Table 4. Six Ioci, Idh-B, Me, Mpi, Pep-D, 
Table 4. Merluccius paradoxus. Allelic mobilities were measured using the most common allele in $M$. capensis for each locus. The following loci were fixed for the 100 allele in all populations: $C k-B, G a p-2, G d a, G p d-A, G p d-B, L d h-C, M d h-A, P e p-X$ (leu-tyr), $P t-1, P t-2, P t-3, P t-4, S d h$. Other loci were fixed for the following alleles: $C k-A(65), N p(110), P t-5(97)$

\begin{tabular}{|c|c|c|c|c|c|c|c|c|c|c|c|}
\hline \multirow[t]{2}{*}{ Locus } & \multirow[t]{2}{*}{ Allele } & \multicolumn{10}{|c|}{ Sample number } \\
\hline & & 1 & 2 & 3 & 4 & 5 & 6 & 7 & 8 & 9 & 10 \\
\hline \multirow[t]{2}{*}{ Gap-1 } & 100 & - & - & - & - & - & 0.006 & - & 0.005 & - & - \\
\hline & 200 & 1.0 & 1.0 & 1.0 & 1.0 & 1.0 & 0,994 & 1.0 & 0.995 & 1.0 & 1.0 \\
\hline \multirow[t]{2}{*}{$G p d-C$} & 100 & 0.008 & - & - & 0.006 & - & - & - & - & - & - \\
\hline & 90 & 0.992 & 1.0 & 1.0 & 0.994 & 1.0 & 1.0 & 1.0 & 1.0 & 1.0 & 1.0 \\
\hline \multirow[t]{9}{*}{$G p i-A$} & 800 & - & - & - & 0.015 & - & - & - & - & - & - \\
\hline & 700 & - & - & - & - & - & - & - & - & 0.005 & - \\
\hline & 400 & 0.017 & 0.020 & 0.020 & 0.020 & 0.020 & 0.018 & 0.032 & 0.005 & 0.025 & 0.022 \\
\hline & 350 & - & - & - & 0.030 & 0.010 & - & 0.005 & - & - & - \\
\hline & 50 & 0.983 & 0.974 & 0.966 & 0.945 & 0.955 & 0.976 & 0.957 & 0.995 & 0.970 & 0.946 \\
\hline & -100 & - & 0.005 & 0.007 & 0.005 & - & - & 0.005 & 0.005 & - & 0.033 \\
\hline & -350 & - & - & - & - & - & 0.006 & - & - & - & - \\
\hline & -700 & - & - & 0.007 & - & - & - & - & - & - & - \\
\hline & $2 \mathrm{~N}$ & 178 & 196 & 148 & 200 & 198 & 164 & 188 & 196 & 200 & 92 \\
\hline \multirow[t]{4}{*}{$G p i-B$} & 90 & 0.006 & - & - & - & - & - & 0.005 & 0.010 & 0.005 & 0.033 \\
\hline & 83 & 0.989 & 1.000 & 0.993 & 0.995 & 1.000 & 1.000 & 0.989 & 0.985 & 0.995 & 0.967 \\
\hline & 60 & 0.006 & - & 0.007 & 0.005 & - & - & - & 0.005 & 0.005 & - \\
\hline & $2 \mathrm{~N}$ & 178 & 196 & 148 & 200 & 198 & 164 & 188 & 196 & 200 & 92 \\
\hline \multirow[t]{4}{*}{$I d h-A$} & 100 & 0.207 & 0.144 & 0.152 & 0.175 & 0.199 & 0.116 & 0.172 & 0.155 & 0.121 & 0.207 \\
\hline & 80 & 0.741 & 0.856 & 0.826 & 0.760 & 0.786 & 0.872 & 0.817 & 0.845 & 0.869 & 0.772 \\
\hline & 40 & 0.052 & - & 0.022 & 0.065 & 0.015 & 0.012 & 0.011 & - & 0.010 & 0.022 \\
\hline & $2 \mathrm{~N}$ & 174 & 194 & 138 & 200 & 196 & 164 & 186 & 194 & 198 & 92 \\
\hline \multirow[t]{4}{*}{$L d h-A$} & 1000 & 0.006 & 0.005 & 0.020 & 0.010 & 0.025 & 0.018 & 0.016 & 0.010 & 0.015 & - \\
\hline & 100 & 0.989 & 0.985 & 0.980 & 0.990 & 0.975 & 0.976 & 0.984 & 0.990 & 0.985 & 1.000 \\
\hline & -1000 & 0.006 & 0.010 & - & - & - & 0.006 & - & - & - & - \\
\hline & $2 \mathrm{~N}$ & 178 & 196 & 148 & 200 & 198 & 164 & 188 & 196 & 200 & 92 \\
\hline \multirow[t]{4}{*}{$L d h-B$} & 100 & 1.000 & 1.000 & 0.953 & 1.000 & 1.000 & 1.000 & 1.000 & 0.995 & 1.000 & 1.000 \\
\hline & 80 & - & - & 0.027 & - & - & - & - & 0.005 & - & - \\
\hline & 70 & - & - & 0.020 & - & - & - & - & - & - & - \\
\hline & $2 \mathrm{~N}$ & 178 & 196 & 148 & 200 & 198 & 164 & 188 & 196 & 200 & 92 \\
\hline \multirow[t]{4}{*}{$M d h-3$} & 120 & - & - & - & - & - & - & - & - & - & 0.043 \\
\hline & 100 & - & - & - & - & - & - & - & - & - & 0.011 \\
\hline & 80 & 1.000 & 1.000 & 1.000 & 1.000 & 1.000 & 1.000 & 1.000 & 1.000 & 1.000 & 0.946 \\
\hline & $2 \mathrm{~N}$ & 178 & 196 & 148 & 200 & 196 & 164 & 188 & 196 & 200 & 92 \\
\hline \multirow[t]{6}{*}{$M e$} & 112 & - & - & - & 0.010 & - & - & - & 0.005 & 0.011 & - \\
\hline & 107 & 0.022 & 0.036 & 0.061 & 0.030 & 0.045 & 0.018 & 0.037 & 0.051 & 0.030 & 0.054 \\
\hline & 100 & 0.978 & 0.949 & 0.919 & 0.930 & 0.934 & 0.970 & 0.957 & 0.908 & 0.940 & 0.902 \\
\hline & 95 & - & 0.010 & 0.020 & 0.025 & 0.015 & 0.012 & 0.005 & 0.020 & 0.020 & 0.033 \\
\hline & 90 & - & 0.005 & - & 0.005 & - & - & - & 0.015 & 0.005 & - \\
\hline & $2 \mathrm{~N}$ & 178 & 196 & 148 & 200 & 198 & 164 & 188 & 196 & 200 & 92 \\
\hline \multirow[t]{9}{*}{$M p i$} & 108 & - & - & - & - & - & - & 0.006 & 0.006 & - & - \\
\hline & 104 & - & - & 0.007 & 0.010 & 0.020 & - & - & 0.005 & - & - \\
\hline & 102 & 0.135 & 0.146 & 0.139 & 0.177 & 0.173 & 0.146 & 0.114 & 0.165 & 0.120 & 0.076 \\
\hline & 100 & - & - & - & - & - & 0.006 & - & 0.015 & - & - \\
\hline & 97 & 0.725 & 0.620 & 0.604 & 0.636 & 0.617 & 0.604 & 0.625 & 0.655 & 0.705 & 0.826 \\
\hline & 95 & - & - & 0.014 & 0.005 & 0.005 & 0.043 & 0.017 & - & 0.015 & - \\
\hline & 93 & 0.129 & 0.229 & 0.222 & 0.162 & 0.184 & 0.171 & 0.205 & 0.170 & 0.145 & 0.087 \\
\hline & 87 & 0.011 & 0.005 & 0.014 & 0.010 & - & 0.024 & 0.034 & 0.005 & - & 0.011 \\
\hline & $2 \mathrm{~N}$ & 178 & 192 & 144 & 198 & 196 & 164 & 176 & 194 & 200 & 92 \\
\hline \multirow[t]{5}{*}{$P g d$} & 65 & - & - & - & - & - & 0.006 & - & - & - & - \\
\hline & 62 & 0.011 & - & - & - & - & - & - & - & - & - \\
\hline & 60 & 0.989 & 0.969 & 1.000 & 1.000 & 1.000 & 0.994 & 1.000 & 1.000 & 1.000 & 1.000 \\
\hline & 40 & - & 0.031 & - & - & - & - & - & - & - & - \\
\hline & $2 \mathrm{~N}$ & 178 & 196 & 148 & 200 & 198 & 164 & 188 & 196 & 200 & 92 \\
\hline
\end{tabular}


Table 4 (continued)

\begin{tabular}{|c|c|c|c|c|c|c|c|c|c|c|c|}
\hline \multirow[t]{2}{*}{ Locus } & \multirow[t]{2}{*}{ Allele } & \multicolumn{10}{|c|}{ Sample number } \\
\hline & & 1 & 2 & 3 & 4 & 5 & 6 & 7 & 8 & 9 & 10 \\
\hline \multirow[t]{7}{*}{$P e p-D$} & 110 & - & - & 0.007 & 0.010 & - & 0.006 & 0.016 & - & 0.005 & - \\
\hline & 108 & 0.265 & 0.240 & 0.215 & 0.210 & 0.176 & 0.213 & 0.231 & 0.199 & 0.230 & 0.217 \\
\hline & 103 & 0.571 & 0.547 & 0.542 & 0.540 & 0.596 & 0.494 & 0.532 & 0.591 & 0.515 & 0.555 \\
\hline & 100 & - & 0.010 & - & - & 0.005 & 0.018 & 0.005 & 0.005 & - & - \\
\hline & 98 & 0.165 & 0.203 & 0.236 & 0.235 & 0.223 & 0.268 & 0.215 & 0.204 & 0.245 & 0.228 \\
\hline & 85 & - & - & - & 0.005 & - & - & - & - & 0.005 & - \\
\hline & $2 \mathrm{~N}$ & 170 & 192 & 144 & 200 & 188 & 164 & 186 & 186 & 200 & 92 \\
\hline \multirow[t]{8}{*}{$P g_{n}-A$} & 150 & 0.006 & - & 0.007 & 0.005 & - & - & 0.005 & - & - & - \\
\hline & 140 & 0.057 & 0.046 & 0.034 & 0.050 & 0.030 & 0.055 & 0.032 & 0.011 & 0.045 & 0.022 \\
\hline & 130 & - & - & - & - & - & 0.006 & 0.005 & 0.005 & - & - \\
\hline & 120 & 0.989 & 0.903 & 0.939 & 0.900 & 0.914 & 0.890 & 0.936 & 0.888 & 0.930 & 0.913 \\
\hline & 100 & - & - & 0.007 & - & - & - & 0.005 & - & 0.005 & 0.011 \\
\hline & 97 & 0.034 & 0.051 & 0.014 & 0.045 & 0.051 & 0.043 & 0.011 & 0.066 & 0.010 & 0.054 \\
\hline & 77 & 0.066 & - & - & - & 0.005 & 0.006 & 0.005 & - & 0.010 & - \\
\hline & $2 \mathrm{~N}$ & 176 & 196 & 148 & 200 & 198 & 164 & 188 & 196 & 200 & 92 \\
\hline \multirow[t]{5}{*}{$P g m-B$} & 102 & 0.006 & - & 0.014 & 0.005 & 0.010 & 0.006 & 0.005 & - & 0.040 & - \\
\hline & 97 & 0.972 & 0.908 & 0.953 & 0.910 & 0.919 & 0.951 & 0.931 & 0.949 & 0.885 & 0.935 \\
\hline & 95 & 0.022 & 0.092 & 0.034 & 0.080 & 0.071 & 0.043 & 0.064 & 0.051 & 0.075 & 0.065 \\
\hline & 92 & - & - & - & 0.005 & - & - & - & - & - & - \\
\hline & $2 \mathrm{~N}$ & 178 & 196 & 148 & 200 & 198 & 164 & 188 & 196 & 200 & 92 \\
\hline \multirow[t]{4}{*}{ Sod } & -100 & 0.978 & 0.995 & 1.000 & 0.995 & 0.990 & 0.988 & 1.000 & 0.990 & 0.990 & 0.989 \\
\hline & -500 & 0.022 & 0.005 & - & 0.005 & 0.010 & 0.006 & - & 0.010 & 0.010 & 0.011 \\
\hline & -1000 & - & - & - & - & - & 0.006 & - & - & - & - \\
\hline & $2 \mathrm{~N}$ & 178 & 196 & 148 & 200 & 198 & 164 & 188 & 196 & 200 & 92 \\
\hline
\end{tabular}

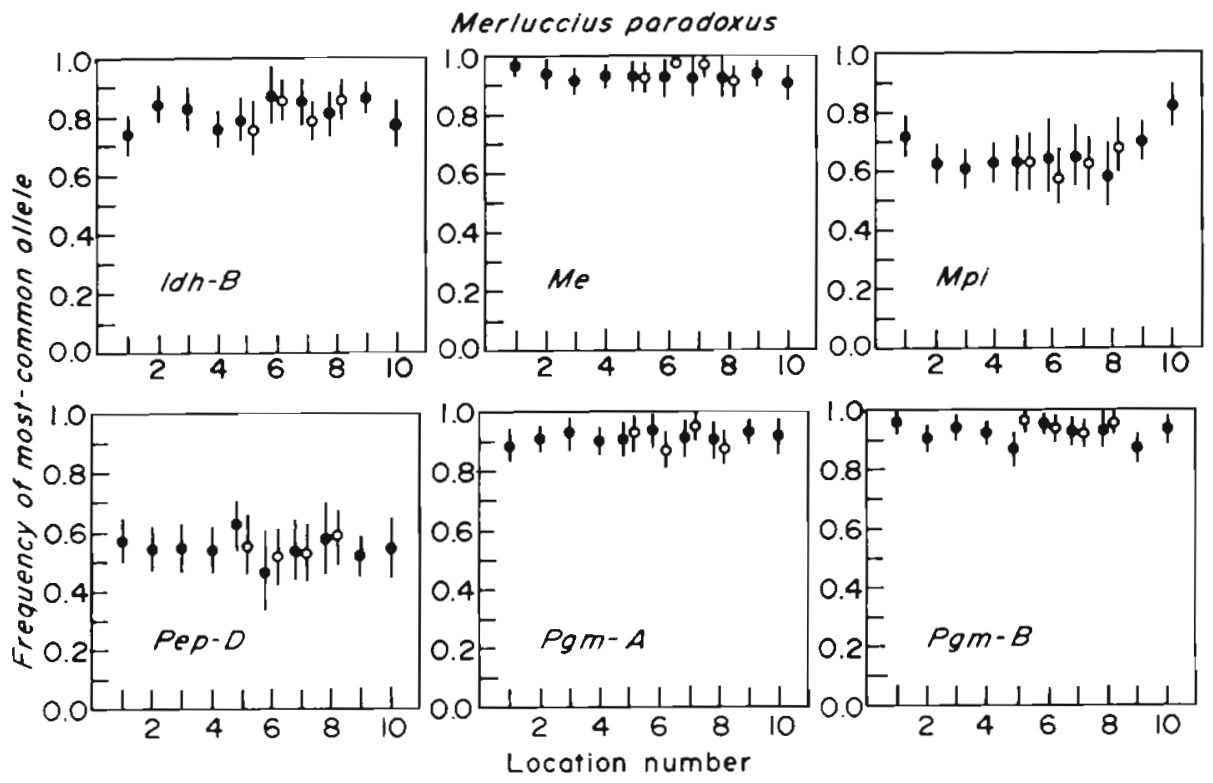

Fig. 3. Frequencies of most common alleles in samples of Merluccius paradoxus. (•) Frequencies of males; $(0)$ frequencies of females. Vertical bars represent 4 binomial standard errors and approximate a $96 \%$ confidence interval. Location numbers as in Table 1 and Fig. 1

Pgm-A, and Pgm-B, were sufficiently polymorphic for statistical analysis (Fig. 3). Individuals were sexed in Samples 5 to 8. Significant departures from HardyWeinberg expectations were detected for $I d h-B$ among males in Sample $8\left(G_{1}=3.92, p<0.05\right)$ and among pooled individuals in the same sample $\left(G_{1}=5.52\right.$, $\mathrm{p}<0.05)$. Significance was detected for $L d h-B$ in Sam- ple $3\left(G_{1}=8.89, \mathrm{p}<0.01\right)$, for $M p i$ in Sample 7 among individuals pooled over sexes $\left(G_{1}=4.39, \mathrm{p}<0.05\right)$. Significant departures were also observed for $P g m-B$ in Sample $4\left(G_{1}=12.07, \mathrm{p}<0.01\right)$ and among males in Sample $5\left(G_{1}=5.12, \mathrm{p}<0.05\right)$.

A single significant difference between sexes was detected for Pgm-B $\left(G_{1}=6.92, p<0.055\right)$. Significant 
allele-frequency heterogeneity was detected among locations in South Africa for Mpi $\left(G_{8}=19.15\right.$; $\left.\mathrm{p}<0.05\right)$. This significance appears to be due to an aberrant frequency in the eastern-most sample. There were no significant differences for the other enzymes and the sum of $G$ over loci was not significant. A significant degree of allele-frequency heterogeneity was also detected among Namibian samples for Idh-B $\left(G_{4}=\right.$ 10.03, $\mathrm{p}<0.05)$ and for Pgm-B $\left(G_{4}=10.22, \mathrm{p}<0.05\right)$. The sum of $G$ over loci for this comparison was also significant $\left(G_{32}=49.54, \mathrm{p}<0.05\right)$. The between-regions comparison was significant for $I d h-B\left(G_{1}=6.99\right.$, $\mathrm{p}<0.01$ ), but the summed value of $G$ was not.

A slightly different picture emerges using approximate $F$ ratios to test for heterogeneity at the different nested levels. These results suggest that the degree of heterogeneity among the Namibian samples $\left(F_{32,8}=\right.$ $1.46, \mathrm{~ns})$ and that among South African samples $\left(F_{23,24}\right.$ $=1.57$, ns) was no greater than the genetic heterogeneity between sexes within each sample. The results further indicated that the degree of allele-frequency heterogeneity between regions was no greater than that among samples collected within each region $\left(F_{8,64}=0.82, \mathrm{~ns}\right)$.

The gene diversity analysis showed that sample heterozygosities ranged from 0.059 to 0.068 and averaged 0.065 . This represented $98.9 \%$ of the total genetic variability on average. Allele-frequency differences between sexes represented $0.3 \%$, differences among samples within regions represented $0.7 \%$ and differences among regions represented $0.1 \%$ of the total genetic variability. The average genetic distance between pairs of samples was 0.0007 and there was no geographic structure in the distribution of genetic distances among samples.

\section{DISCUSSION}

\section{Within-sample variation}

It is difficult to interpret the significant allele-frequency differences that we found between the sexes of both hake species. For Merluccius capensis, we made a total of 60 comparisons between sexes over the 6 polymorphic enzymes and found significant differences between sexes at 5 locations. With a significance level set at $p=0.05$ we would expect 3 of these comparisons to be significant by chance alone because of our finite sample sizes. It is still possible, but unlikely, that all 5 significant differences could be within the possibility of sampling error. For $M$. paradoxus, we detected only 2 significant differences between sexes out of 24 comparisons, one of which would be expected by chance alone.
If in fact these allele-frequency differences are not just due to sampling errors, there are 2 possible explanations. The first hypothesis centers on the population biology of these hakes and is that the subsamples were drawn from mixed but genetically differentiated subpopulations of each sex. This hypothesis may have some merit because females grow faster, mature later and live longer than males, and because females are larger they tend to inhabit deeper water than males of the same age (Inada 1981). Such behavior tends to isolated the sexes from one another so that catches from deep waters consist primarily of large females and those from shallower waters consist for the most part of smaller males for each species (pers. comm.; R. Leslie, Sea Fisheries Research Institute). If this hypothesis is true, we might expect to find a significant degree of genetic heterogeneity among samples from different areas and a consistent deficit of heterozygotes in samples pooled between sexes. Neither of these predictions, however, were apparent in our samples. The degree of genetic heterogeneity among samples was small (see later), and there were no consistent deficits of heterozygotes in samples pooled between sexes.

The second possibility is that some kind of sexspecific selection is operating. Since large mature females inhabit the deepest water, there may be environmental factors such as hydrostatic pressure, temperature or environmental heterogeneity which act as selective forces on various genotypes (e.g. Hilbish et al. 1982, Siebenaller et al. 1982). One selective model that has received much attention is heterozygote advantage in which we might expect to find an excess of heterozygotes at loci under selection, i.e. those loci showing significant allele-frequency differences between sexes. This was not the case, however, for Merluccius capensis. None of the samples showing significant differences between sexes showed a significant departure from Hardy-Weinberg expectations or a consistent excess (or deficit) of heterozygotes. Another possibility is that there is some kind of directional selection operating on fish found in different habitats. In such a model, we would expect to find that the frequency of one allele or another is consistently greater in one of the sexes. This, however, was also not apparent in the allele frequency data (Fig. 2).

Significant allele frequency differences between sexes in the same sample have also been reported for 2 other marine fishes. Mork et al. (1985) found significant differences for 4 enzymes in Atlantic cod Gadus morhua at 2 locations. The enzymes showing the significant differences, however, did not deviate significantly from Hardy-Weinberg expectations and there was no obvious explanation for the sex differences. In another study, significantly different allele frequencies between sexes were observed for witch flounder Glyp- 
tocephalus cynoglossus in a single sample located in the Gulf of St. Lawrence, Canada (Fairbairn 1981). This difference was accompanied by a significant heterozygote deficit and Fairbairn (1981) postulated that the difference between sexes had arisen by selection for rare alleles in females. Alternatively, the difference may have arisen by chance because no other significant differences between sexes were observed in that study. Without applying ad hoc explanations to individual samples, there does not seem to be a general explanation for allele frequency differences between sexes, a not infrequent occurrence in marine fishes.

\section{Geographic variation}

Marine fishes in general tend to show very little genetic subdivision between geographic stocks (e.g. Ryman et al. 1984, Shaklee 1984) because there is a very large potential for gene flow between areas by the passive drift of larvae in ocean currents and by active migration of adults. Gene flow, when it occurs, acts as a strong homogenizing force on geographically separated stocks or populations (Waples 1987). Another reason that marine fishes are characterized by a general lack of population subdivision is that genetic drift is negligible at the very large population sizes that are typical of marine fishes. Even for populations that become completely isolated from one another, at least $N(=$ population size) generations must pass before any substantial divergence is likely (Kimura 1955). Nonetheless, genetic subdivisions among regional stocks of marine fishes have been reported, which appear to be the result of ancient allopatric subdivision rather than the result of differentiation in situ (Grant 1987).

Our allozyme data show that there is only a small amount of genetic divergence between the stocks of each species of hake. Genetic distances between samples were remarkably small, generally less than 0.001 , and more than $98 \%$ of the total genetic diversity on average was found within locations for both species. Nonetheless, there was a significant degree of allele frequency heterogeneity between the Namibian and South African stocks of Merluccius capensis which appear in both the nested contingency-table analysis and the analysis using approximate $F$-ratios. Most of the regional heterogeneity, about $80 \%$ of the G-statistic summed over loci, was due to regional differences in the allele frequencies of $C k-A$. None of the other polymorphic loci showed the same degree of geographic differentiation for this comparison.

It is difficult to account for this regional heterogeneity. Assuming that allozyme variation is largely neutral to natural selection and that the geographic distributions of alleles are due to population events, one explanation is that ancestral populations of hake were geographically subdivided and isolated in some way. There were several episodes of oceanic cooling in the Pleistocene which may have subdivided and displaced populations of hake northward to either side of the southern African subcontinent. A similar biogeographic model appears to explain genetic subdivision between eastern and western populations of several North Pacific Ocean fishes in which coastal Pleistocene glaciation and oceanic cooling in the central North Pacific Ocean apparently acted as a barrier to migration isolating eastern and western populations (Grant et al. 1983, Grant \& Utter 1984, Grant et al. 1987). In Pacific herring, for example, the average genetic distance between the 2 major groups was 0.039 (40 loci), whereas the average genetic distance between locations within groups was only 0.003 . The small amount of overall divergence between the groups of hake suggest that this explanation is unlikely.

If the regional differentiation in Merluccius capensis was due to historical population events we would expect, first of all, that all of the loci in a species would be affected to the same extent and that each locus would show a similar amount of divergence (Allendorf \& Phelps 1981). Our observation that $C k-A$ shows a significantly greater degree of geographic differentiation than other loci suggests that this locus may be under selection. It is, however, notoriously difficult to make a formal test of a selective hypothesis with allelefrequency data alone (Ewens \& Feldman 1976). Secondly, if paleo-oceanographic events subdivided populations of one species of fish in southern Africa, then they would have similarly affected other species in the same area. Genetic studies of southern African anchovies Engraulis capensis (Grant 1985a), pilchards Sardinops ocellata (Grant 1985b) and M.paradoxus, however, do not show corresponding degrees of genetic subdivision over this area.

Our genetic results are somewhat ambiguous over the question of whether Namibian and South African hakes represent 1 or 2 stocks. Ihssen et al. (1981) point out that 'observed electrophoretic difference is a sufficient but not necessary condition for 2 groups of fish to be genetically differentiated' and suggest that population, morphometric and meristic data also be used to infer stock structure. There are minor morphological differences between Namibian and South African stocks of both species. North of the Orange River, the anal fin of Merluccius capensis is entirely white and that of M. paradoxus is black in color, and this difference is used by research biologists to separate species in the field (E. Macpherson, Instituto de Investigaciones Pesqueras, Barcelona). In this area, this difference between species consistently parallels other differences such as gill tubercle (van Eck 1969) and otolith 
morphology (Botha 1971). At the Orange River and to the south, however, both species tend to have gray anal fins with black edges. In this southern area, gill arch tubercle morphology is used to identify species. The geographic variation in anal fin color may reflect genetic differences not detectable with electrophoretic methods or may reflect environmental influences on a genotype capable of variable expression. Either interpretation suggests that there is at least a small degree of regional subdivision between these 2 stocks.

Many species of hake show seasonal longshore migrations driven by annual cycles of cooling and warming. Generally hakes migrate poleward during summer and toward the equator in winter (Inada 1981, Bailey et al. 1982). Such migratory systems should of course be managed as a single panmictic population over the whole geographic range of migration. The extent of longshore migration in the southern African hakes appears to vary from region to region. There have been no tagging studies to investigate patterns of migration because hakes do not survive capture and release well (Fritz 1959). Therefore we can only infer migrational patterns from seasonal and geographic variation in density. An analysis of densities by age show that the greatest concentrations of adult fishes for Merluccius capensis and $M$. paradoxus tend to be located off Cape Point and off the mouth of the Orange river (Payne et al. 1986). The densities of younger fishes, however, tend to be geographically more evenly distributed. The northward flowing Benguela Current on the west coast undoubtedy has an homogeñining effect by passivcly transporting planktonic eggs, larvae and small juveniles. There is no indication of seasonal longshore movements in the concentrations of South African hakes. In contrast, there appears to be at least some seasonal longshore movement of hake stocks in the Namibia region. The greatest concentrations of $M$. capensis tend to be located between $17^{\circ}$ and $19^{\circ} \mathrm{S}$ in summer and autumn but in the vicinity of Walvis Bay $\left(22^{\circ}\right.$ to $\left.24^{\circ} \mathrm{S}\right)$ in late winter and early spring (Anon. 1986). This movement corresponds with seasonal movement of the boundary between the warm Southern Equatorial Current and the cold upwelled Benguela Current (Inada 1981).

We thus conclude that there is restricted movement of adults between Namibian and South African waters. On the other hand, there may be passive northward movement of eggs and larvae by the Benguela Current. Nonetheless, our finding of a small, but measurable, degree of genetic differentiation between Namibian and South African waters for Merluccius capensis suggests that the amount of gene flow between these areas is restricted to at least some degree. These results endorse the present policy of managing Namibian and South African stocks of $M$. capensis as 2 separate units.
Although there was no evidence for genetic subdivision between stocks of $M$. paradoxus, the most conserative approach would also be to treat Namibian and South African stocks as separate units.

Acknowledgements. We are grateful to Dr E. Macpherson and the crew of the M.F.V Chicha Touza, Instituto de Investigaciones Pesqueras, Barcelona, Spain, for the collection of samples in Namibia. C. Hart and the crew of the R. V. Africana assisted with the collection of samples in South African waters. Dr Wessels of the Fishing Industry Research Institute, University of Cape Town, kindly provided laboratory facilities. This work was supported for the most part by the Sea Fisheries Research Institute, Cape Town and by the South African Council of Oceanographic research (S.A.N.C.O.R.).

\section{LITERATURE CITED}

Allendorf, F. W., Phelps, S. R. (1981). Use of allelic frequencies to describe population structure. Can. J. Fish. Aquat. Sci. 38: 1507-1514

Allendorf, F. W., Utter, F. M. (1979). Population genetics. In: Hoar, W. S., Randall, D. J., Brett, J. R. (ed.) Fish physiology, Vol. 8. Academic Press, New York, p. 407-454

Anonymous (1986). Report of the standing committee on stock assessement (STOCK). In: Proceedings and Reports of Meetings 1985, Part II. Int. Comm. SE Atl. Fish., Madrid, Spain, p. 51-95

Bailey, K. M., Francis, R. C., Stevens, P. R. (1982). The life history and fishery of Pacific whiting, Merluccius productus. Calif. Coop. Oceanic Fish. Invest. Rep. 23: 81-98.

Becker, I. I., Grant, W. S., Kirby, R. W., Robb, F. T. (1988). Evolutionary divergence between sympatric species of southern African hakes, Merluccius capensis and $M$. paradoxus. 1. Restriction enzyme analysis of mitochundrial DNA. Heredity (in press)

Bentz, K. L. M. (1976). Gill arch morphology of the Cape hakes, Merluccius capensis Cast. and M. paradoxus Franca. Fish. Bull. S. Afr. 8: 17-22

Booke, H. E. (1981). The conundrum of the stock concept-are nature and nurture definable in fishery science? Can. J. Fish. Aquat. Sci. 38: 1479-1480

Botha, L. (1971). Growth and otolith morphology of the Cape hakes, Merluccius capensis Cast. and $M$. paradoxus Franca. Investl Rep. Div. Sea Fish. Un. S. Afr. 97: 1-32

Botha, I. (1973). Migrations and spawning behaviour of the Cape hakes. S. Afr. Shipp. News Fish. Indust. Rev. 28: 62-67

Botha, L. (1980). The biology of the Cape hakes Merluccius capensis Cast. and $M$. paradoxus Franca in the Cape of Good Hope area. Ph. D. thesis, Univ. Stellenbosch, S. Africa

Chakraborty, R., Haag, M., Ryman, N., Ståhl, G. (1982). Hierarchical gene diversity analysis and its application to brown trout population data. Hereditas 97: 17-22

Ewens, W. J., Feldman, M. W. (1976). The theoretical assessment of selective neutrality. In: Karlin, S., Nevo, E. (ed.) Population genetics and ecology. Academic Press, New York, p. 303-338

Fairbairn, D. J. (1981). Which witch is which? A study of the stock structure of witch flounder (Glyptocephalus cynoglossus) in the Newfoundland Region. Can. J. Fish. Aquat. Sci. 38: 782-794

Fisher, S. E., Shaklee, J. B., Ferris, S. D., Whitt, G. S. (1980). Evolution of five multilocus isozyme systems in chordates. Genetica 52/53: 73-85 
Franca, P. (1954). Contribuicao para o conhecimento do genero Merluccius no Atlantico oriental ao sul do equador. Trabhs. Miss. Biol. Marit. 8: 46-98

Frick, L. (1983). An electrophoretic investigation of the cytosolic di- and tripeptidases of fish: molecular weights, substrate specificities, and tissue and phylogenetic dis. tributions. Biochem. Genet. 21: 309-322

Fritz, R. L. (1959). Hake tagging in Europe and the United States, 1931-1958. J. Cons. int. Explor. Mer 24: 480-485

Grant, W. S. (1985a). Biochemical genetic stock structure of the southern African anchovy, Engraulis capensis Gilchrist. J. Fish Biol. 27. 23-29

Grant, W.S. (1985b). Population genetics of the southern African pilchard, Sardinops ocellata, in the Benguela upwelling system. In: Bas, C., Margalef, R, Rubies, P. (ed.) Simposio internacional sobre las areas de afloramiento mas importantes del oeste Afriano. Invest. pesq., Barcelona, p. 551-562

Grant, W. S. (1987). Genetic divergence between congeneric Atlantic and Pacific Ocean fishes. In: Ryman, N., Utter, F. (ed.) Population genetics and fishery management. Univ Washington Press, Seattle, p. 225-246

Grant, W. S., Utter, F. M. (1984). Biochemical population genetics of Pacific herring (Clupea pallasi). Can. J. Aquat. Sci. 41: 856-864

Grant, W. S., Bakkala, R., Utter, F, M., Teel, D. J., Kobayashi, T. (1983). Biochemical genetic population structure of yellowfin sole, Limanda aspera, of the North Pacific Ocean and Bering Sea. Fish. Bull. U.S. 81: 667-677

Grant, W. S., Becker, I. I., Leslie, R. W. (1988). Evolutionary divergence between sympatric species of southern African Hakes Merluccius capensis and $M$. paradoxus. I. Electrophoretic analysis of proteins. Heredity (in press)

Grant, W. S., Zhang, I. C., Kobayashi, T., Stahl, G. (1987). Lack of genetic stock discretion in Pacific cod (Gadus macrocephalus). Can. J. Fish. Aquat. Sci. 44: 490-498

Haugen, C. W., Messersmith, J. D., Wickwire, R. H. (1969). Progress report on anchovy tagging off California and Baja California, March 1966 through May 1969. Fish. Bull. Calif. 147: 75-86

Hilbish, T. J., Deaton, L. E., Koehn, R. K. (1982). Effect of an allozyme polymorphism on regulation of cell volume. Nature, Lond. 298: 688-689

Hourston A. S. (1982). Homing by Canada's west coast herring to management units and divisions as indicated by tag recoveries. Can. J. Fish. Aquat. Sci. 39: 1414-1422

חles, T. D., Sinclair, M. (1982). Atlantic herring stock discreteness and abundance. Science 215: 627-633

Inada, T. (1981). Studies on the merlucciid fishes. Bull. Far Seas Fish. Res. Lab., Shimizu, Japan 18: 1-172

Ihssen, P. E., Booke, H. E., Casselman, J. M., McGlade, J. M., Payne, N. R., Utter, F. M. (1981). Stock identification: materials and methods. Can. J. Fish. Aquat. Sci. 38 1838-1855

Jones, B. W. (1974). World resources of hakes of the genus Merluccius. In: Harden Jones, F. R. (ed.) Sea fisheries research. John Wiley \& Sons, New York, p. 139-166

Jones, B. W., Mackie, I. M. (1970). On application of electrophoretic analysis of muscle myogens to taxonomic studies in the genus Merluccius. Comp. Biochem. Physiol. 32: $267-273$

Kimura, M. (1955). Solution of a process of random genetic drift with a continuous model. Proc. natl Acad. Sci. U.S.A. 41: $144-150$
Lewontin, R. C. (1974). The genetic basis of evolutionary change. Columbia University Press, New York

Macpherson, E., Roel, B., Morales, B. (1986). Evolucion del reclutamiento de la merluza y distribucion y abundancia de varias expecies comerciales en 1985 en las Devisiones 1.4 y 1.5. Colln. Scient. Pap. Int. Comm. SE Atl. Fish. 13: 113-136

Mangaly, G., Jamieson, A. (1978). Genetic tags applied to the European hake, Merluccius merluccius (L.). Anim. Blood Grps. Biochem. Genet. 9: 39-48

Markert, C. L., Shaklee, J. B., Whitt, G. S. (1975). Evolution of genetic variation in Atlantic cod (Gadus morhua) throughout its range. Can. J. Fish. Aquat. Sci. 42: 1580-1587

Mork, J., Ryman, N., Ståhl, G., Utter, F., Sundnes, G. (1985). Genetic variation in Atlantic cod (Gadus morhua) throughout its range. Can. J. Fish. Aquat. Sci. 42: 1580-1587

Nei, M. (1972). Genetic distance between populations. Am. Nat. 106: 283-292

Nei, M. (1973). Analysis of gene diversity in subdivided populations. Proc. natl Acad. Sci. U.S.A. 70: 3321-3323

Payne, A. I. L., Augustyn, C. J., Leslie, R. W. (1986). Results of the South African hake biomass cruises in division 1.6 in 1985 Colln Scient. Pap. Int. Comm. SE Atl. Fish. 13: 181-196

Ricker, W. E. (1972). Heredity and environmental factors affecting certain salmonid populations. In: Simon, R. C., Larkin, P. A. (ed.) The stock concept in Pacific salmon. MacMillan Lect. Fish., University of British Columbia, Canada, p. 19-160

Ryman, N., Lagercrantz, U., Anderson, L., Chakraborty, R., Rosenberg, R. (1984). Lack of correspondence between genetic and morphologic variability patterns in Atlantic herring (Clupea harengus). Heredity 53: 687-704

Shaklee, J. B. (1984). Genetic variation and population structure in the damselfish, Stegastes fasciolatus, throughout the Hawaiian Archipelago. Copeia 1984 (3): 629-640

Shannon, L. V. (1985). The Benguela ecosystem, Part I. Evolution of the Benguela physical features and processes. Oceanogr. mar. Biol. A. Rev. 23: 105-182

Siebenaller, J. F., Somero, G. N., Haedrich, R. L. (1982) Biochemical characteristics of macrourid fishes differing in their depths of distribution. Biol. Bull. mar. biol. Lab. Woods Hole 163: 240-249

Smouse, P. E., Kojima, K. I. (1972). Maximum likelihood analysis of population differences in allelic frequencies. Genetics 72: 709-719

Sneath, P. H. A., Sokal, R. R. (1973). Numerical taxonomy. Freeman, San Francisco

Sokal, R. R., Rohlf, R. J. (1981). Biometry, 2nd edn. Freeman and Co., San Francisco

Swofford, D. L., Selander, R. B. (1981\}. BIOSYS-1: a FORTRAN program for the comprehensive analysis of electrophoretic data in population genetics and systematics. J. Heredity 72: 281-283

Utter, F. M. Hodgins, H. O. (1959). Lactate dehydrogenase isozymes of Pacific hake (Merluccius productus). J. exp. Zool. 172: 59-68

Utter, F. M. (1971). Biochemical polymorphisms in the Pacific hake (Merluccius productus). Int. Counc. Explor. Sea 161. $87-89$

Utter, F. M., Stormont, C. J., Hodgins, H. O. (1970). Esterase polymorphism in vitreous fluid of Pacific hake, Merluccius productus. Anim. Blood Grps. Biochem. Genet. 1: 69-82

van Eck, T. H. (1969). The South African hake: Merluccius capensis'or Merluccius paradoxus? S. Afr. Shipp. News and Fish. Ind. Rev. 24: 95-97

Waples, R. S. (1987). A multispecies approach to the analysis of gene flow in marine shore fishes. Evolution 41: 385-400 